\title{
Genome-scale data resolve ancestral rock- inhabiting lifestyle in Dothideomycetes (Ascomycota)
}

\author{
Claudio G. Ametrano ${ }^{1 *}$, Felix Grewe ${ }^{2}$, Pedro W. Crous ${ }^{3}$, Stephen B. Goodwin ${ }^{4}$, Chen Liang ${ }^{5}$, Laura Selbmann ${ }^{6,7}$, \\ H. Thorsten Lumbsch'², Steven D. Leavitt ${ }^{8}$ and Lucia Muggia ${ }^{1 *}$ (D)
}

\begin{abstract}
Dothideomycetes is the most diverse fungal class in Ascomycota and includes species with a wide range of lifestyles. Previous multilocus studies have investigated the taxonomic and evolutionary relationships of these taxa but often failed to resolve early diverging nodes and frequently generated inconsistent placements of some clades. Here, we use a phylogenomic approach to resolve relationships in Dothideomycetes, focusing on two genera of melanized, extremotolerant rock-inhabiting fungi, Lichenothelia and Saxomyces, that have been suggested to be early diverging lineages. We assembled phylogenomic datasets from newly sequenced (4) and previously available genomes (238) of 242 taxa. We explored the influence of tree inference methods, supermatrix vs. coalescent-based species tree, and the impact of varying amounts of genomic data. Overall, our phylogenetic reconstructions provide consistent and well-supported topologies for Dothideomycetes, recovering Lichenothelia and Saxomyces among the earliest diverging lineages in the class. In addition, many of the major lineages within Dothideomycetes are recovered as monophyletic, and the phylogenomic approach implemented strongly supports their relationships. Ancestral character state reconstruction suggest that the rock-inhabiting lifestyle is ancestral within the class.
\end{abstract}

Keywords: Lichenothelia, Phylogenomics, Saxomyces, Species tree, Supermatrix, Supertree

\section{INTRODUCTION}

Dothideomycetes is the largest and most diverse fungal class of ascomycetes, comprised of c. 20,000 species (Jaklitsch et al. 2016) classified into 105 families (Hyde et al. 2013) and 32 orders (Liu et al. 2017). The class encompasses great variation of fungal lifestyles, including saprotrophs, plant pathogens, endophytes, epiphytes, fungicolous, lichenized, lichenicolous, and free-living rock-inhabiting fungi.

Several phylogenetic inferences have been generated to resolve taxonomy and evolutionary relationships within Dothideomycetes at different systematic levels (e.g. Schoch et al. 2006, Schoch et al. 2009; Nelsen et al. 2009; Ruibal et al. 2009; Hyde et al. 2013; Muggia et al. 2015; Liu et al. 2017; Ametrano et al. 2019). These analyses usually considered wide taxon sampling and were based on combinations

\footnotetext{
*Correspondence: claudiogennaro.ametrano@phd.units.it; Imuggia@units.it ${ }^{1}$ Department of Life Sciences, University of Trieste, via Giorgieri 10, 34127 Trieste, Italy

Full list of author information is available at the end of the article
}

of nuclear, mitochondrial, and protein-coding loci. However, previous studies inferred inconsistent placement of some orders or families, particularly among the basal nodes. In spite of the increased use of genome-scale data to resolve long-standing evolutionary and taxonomic issues (Chan and Ragan 2013), phylogenomic approaches are rather uncommon for Dothideomycetes, although about 250 sequenced genomes of its representatives have been sequenced. Within this class, genome sequencing efforts have largely focused on plant and human pathogenic fungi (Hane et al. 2007; Ohm et al. 2012; Raffaele and Kamoun2012), and fungi with a certain ecological (e.g., melanized, halotolerant yeast; Gostinčar et al. 2011) or economical interest (e.g. carbohydrate degraders; Prenafeta-Boldu et al. 2006; Sterflinger 2006; Nai et al. 2013). On the other hand, the most inconspicuous taxa have been largely neglected in genomic research - especially those belonging to the group of melanized, meristematic, rock-inhabiting fungi (RIF). Only recently 
few Antarctic RIF genomes have become available (Coleine et al. 2017). This shortcoming is likely due to the difficulty to retrieve those species in nature, isolate them axenically in vitro and their extremely slow growth rate in culture.

Two dothidealean genera, Lichenothelia and Saxomyces, are iconic representatives of RIF (Muggia et al. 2015; Selbmann et al. 2014; Ametrano et al. 2019). Species of Lichenothelia and Saxomyces are widespread worldwide, occurring on exposed rocks, often in extreme environments, and having evolved lifestyles on nutrient-poor substrates. Because they can survive in harsh environments characterized by high solar radiation, very high and very low temperatures, and drought stress, they have been recognized within the group of polyextremotolerant fungi (Gostinčar et al. 2012). Lichenothelia species are of particular interest because they exhibit a multiplicity of lifestyles, e.g. non-lichenized rock-inhabiting, parasitic on lichens, and loosely associated with green algae on rocks. Due to its affinity towards algae, Lichenothelia has been historically considered an evolutionary link between the non-lichenized Dothideomycetes and the lichenized Lecanoromycetes (Hawksworth 1981; Muggia et al. 2013). Recent phylogenetic analyses have identified Lichenothelia and Saxomyces as two individually monophyletic lineages, but their phylogenetic placement within Dothideomycetes remained unresolved (Ametrano et al. 2019). Therefore, more information from Lichenothelia and Saxomyces genomes is required to better understand their genetic diversity and evolutionary relationships with other closely related dothideomycetous taxa with varying lifestyles.

Here, we present a phylogenomic study on the evolutionary relationships of Lichenothelia and Saxomyces within Dothideomycetes. Genome-scale data from de novo genome assemblies of two species of Lichenothelia and two of Saxomyces were added to a supermatrix including genes of most Dothideomycetes taxa for which whole-genome data were available. Our study aimed to (i) generate a genome-scale phylogeny of Dothideomycetes to resolve the phylogenetic placement of still unsupported lineages and in particular clarify that of Lichenothelia and Saxomyces and their relationships with other RIF lineages within the class, (ii) assess whether and to which extent the amount of genetic information, the alignment processing and the phylogenomic reconstruction method impact the final phylogenetic inference, and (iii) assess the minimum amount of genomic information needed to generate a topology that agrees with the phylogeny generated with the entire set of genes.

\section{MATERIALS AND METHODS}

\section{Cultured strains, DNA extraction and sequencing}

Fungal strains representing Lichenothelia and Saxomyces species were available from previous culture isolations reported by Muggia et al. (2013, 2015), Selbmann et al. (2014) and Ametrano et al. $(2017,2019)$. The strains for genome sequencing are: Lichenothelia convexa L1844 (LMCC0061, MUT5682); Lichenothelia intermixta L2282 (LMCC0543); Saxomyces alpinus CCFEE5470 (CBS135222); and Saxomyces americanus L1853 (LMCC0060, MUT5853). Strains were sub-cultured on malt-yeast medium (MY, Ahmadjian 1967) at $20^{\circ} \mathrm{C}$, and DNA was extracted as soon as the mycelia grew to a sufficient biomass (after about 4 weeks).

The fungal biomass was removed from the growth medium, ground in liquid nitrogen and genomic DNA was extracted using the ZR Fungal/Bacterial DNA MicroPrep $^{\text {tw }}$ Kit (Zymo Research) according to the manufacturer's protocol. The quality of the genomic DNA was checked by gel electrophoresis on $0.8 \%$ agarose gel, which showed a sharp genomic DNA band with a small amount of degraded nucleic acid. DNA concentration was 7-11 ng/ $\mathrm{ll}(175-275$ ng of DNA), and the nucLSU rDNA was successfully sequenced to confirm the identity of the strains $(100 \%$ sequence identity). The four genomic DNA extractions were sent to the University of Illinois at Chicago sequencing facility for library preparation (Nextera XT) and sequencing on an Illumina MiSeq platform. The strain of Lichenothelia convexa was sequenced with a coverage three times deeper than the other three strains to obtain a better assembly.

\section{Bioinformatics}

A bioinformatic pipeline consisting of several programs was generated to extract single-copy genes from wholegenome assemblies and create individual gene alignments and phylogenies (Fig. 1). Fastq files containing $2 \times 150$ bp paired-end (PE) reads were quality filtered with Trimmomatic 0.35 (Bolger et al. 2014) to remove sequencing adapters, low-quality nucleotides and short reads. We changed the recommended settings to LEADING:10, TRAILING:10, and MINLEN:25 to trim the ends of the reads when the quality was below 10 and subsequently remove sequences shorter than 25 bases. A quality check was performed with FastQC 0.11.5 both before and after reads trimming. High quality, pairedend and orphan reads were then assembled with SPAdes 3.5.0 using default $\mathrm{k}$-mer lengths based on read length (kmer of 21, 33 and $55 \mathrm{bp}$ for $151 \mathrm{bp}$ reads) (Bankevich et al. 2012). The assemblies from the multi k-mer SPAdes approach were checked with Quality Assessments Tool (QUAST 4.5, Gurevich et al. 2013). The assembly completeness was also analyzed with Benchmarking Universal Single Copy Orthologs (BUSCO 3.0.1; Waterhouse et al. 2017). In addition, 238 whole-genome assemblies of other Dothideomycetes were downloaded from NCBI GenBank and the JGI Genome portal (Additional file 1: Table S1; Galagan et al. 2005; Fedorova et al. 2008; Sharpton et al. 


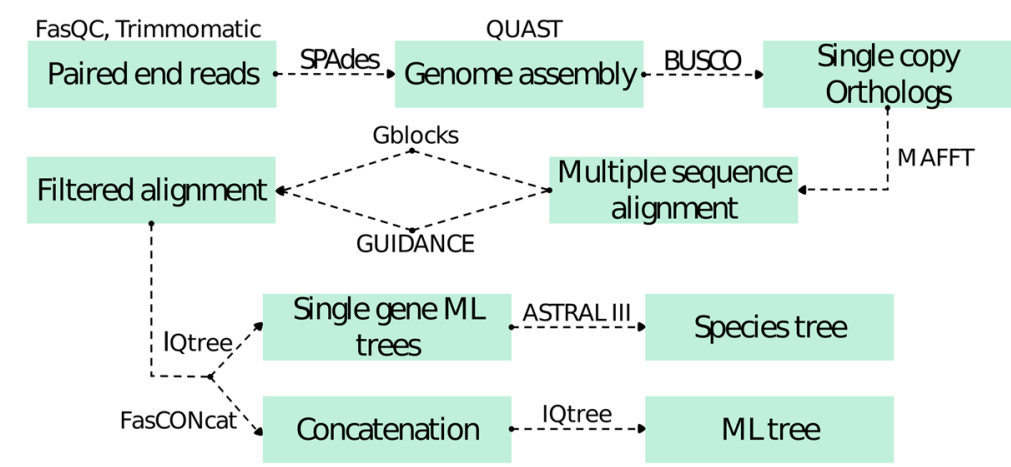

Fig. 1 Flow chart reporting the bioinformatic pipeline used for the analyses. Input and output of the pipeline are reported inside the boxes, the software used for each pipeline step is reported above or between the boxes

2009; Ellwood et al. 2010; Desjardins et al. 2011; Goodwin et al. 2011; Rouxel et al. 2011; Stukenbrock et al. 2011; Chan et al. 2012; Hu et al. 2012; Joardar et al. 2012; $\mathrm{Ng}$ et al. 2012; Ohm et al. 2012; Spatafora et al. 2012; Blanco-Ulate et al. 2013; Condon et al. 2013; Lenassi et al. 2013; Yew et al. 2013; Aragona et al. 2014; Bihon et al. 2014; Chan et al. 2014; Cooke et al. 2014; Gao et al. 2014; Gostinčar et al., 2014; Han et al. 2014; Soliai et al. 2014; Sterflinger et al. 2014; van der Nest et al. 2014; Yang et al. 2014; Franco et al. 2015; Grandaubert et al. 2015; Kuan et al. 2015; MoralesCruz et al. 2015; Orner et al. 2015; Shaw et al. 2015; Shiller et al. 2015; Vaghefi et al. 2015; Wingfield et al. 2015; Bock et al. 2016; Chang et al. 2016; Mosier et al. 2016; Nguyen et al. 2016; Peter et al. 2016; Verma et al. 2016; Wang et al. 2016; Xu et al. 2016; Zeiner et al. 2016; Coleine et al. 2017; Marsberg et al. 2017; Mondo et al. 2017; Shrestha et al. 2017; Teixeira et al. 2017; Zeng et al. 2017; Knapp et al. 2018; Lopez et al. 2018) and processed with BUSCO. All assemblies of Dothideomycetes available during June 2017, when the dataset was built, were included, except those which were taxonomically mis-assigned during test runs of the dataset. Distribution of the BUSCO completeness of the assemblies was assessed for outliers with Thompson Tau test (Thompson 1935). BUSCO evaluation of the completeness of the genome assemblies is based on a set of orthologous genes (OrthoDB; Zdobnov et al. 2016) present in the members of the taxonomic group of interest, Pezizomycotina ortholog gene set was used for Dothideomycetes. Among the genes predicted by BUSCO, only single-copy orthologs, which are suitable for phylogenetic inference, were selected and used for subsequent analyses. Orthologous genes which were present in single copy but predicted in multiple possible versions were also discarded. Selected single-copy genes from each taxon were aligned with MAFFT 7 (Katoh and Standley 2013) using default parameters. As the alignment filtering method can affect the output of the subsequent phylogenetic inferences (Tan et al. 2015), sometimes worsening the phylogenetic results, sequence alignments from MAFFT were filtered either with Gblocks (Castresana 2000) or with GUIDe tree-based AligNment ConfidencE (GUIDANCE 2.02, Penn et al. 2010).

\section{Phylogenomic analyses}

The phylogenetic reliability of the generated dataset was tested with different numbers of genes, alignment filtering methods and tree reconstruction methods. Five individual datasets were constructed: (i) a dataset of genes longer than $1000 \mathrm{bp}$ after Gblocks trimming ("> $1 \mathrm{~kb}$ Gblocks" dataset) and (ii) one after GUIDANCE trimming with less than $50 \%$ of gaps ("> 1 kb GUIDANCE" dataset); (iii) the complete set of retrieved genes including both genes longer and shorter than $1 \mathrm{~kb}$ after Gblocks filtering ("Complete Gblocks" dataset) and (iv) GUIDANCE filtering ("Complete GUIDANCE" dataset); (v) a dataset without any missing data for estimating the impact of missing data on the tree, hence reduced both in gene number and taxa ("No missing" dataset). The individual gene alignments of each dataset were either used for individual gene tree calculations, or concatenated into a supermatrix with FasCONcat 1.0 (Kück and Meusemann 2010) (Fig. 1). Maximum likelihood (ML) phylogenetic inferences from the supermatrix, as well as the single-locus inferences, were produced with IQTree 1.6.1 (Nguyen et al. 2014) using 1000 replicates of ultra-fast bootstrap (-bb) to get node support values (Hoang et al. 2017) and Model Finder Plus (-MFP) to select the most suitable nucleotide substitution model. Gene trees resulting from single-locus inferences were further combined in a supertree with the coalescent-based species tree estimation software ASTRAL III (Zhang et al. 2017). The resulting topologies were compared with normalized Robinson-Foulds distance (RF, Robinson and Foulds 1981).

As the analysis of a genome-based supermatrix with bootstrap support can be highly computationally demanding, an alternative, customized, resampling strategy was tested in the analyses on the "> $1 \mathrm{~kb}$ Gblocks" concatenated alignment. Thirty runs of IQTree were 
carried out on reduced concatenated matrices made of an increasing, randomly selected number of columns from this alignment. The phylogeny resulting from the complete supermatrix was taken as reference and used to compute RF distances in RAxML 8.2 (Stamatakis 2014) with the phylogenies generated from the re-sampled alignments. Resampling was performed without replacement and the sampling effort was increased until no statistically significant difference among RF distance distributions was detected (one-way ANalysis Of Variance (ANOVA) $p<0.01$ and post hoc pairwise tests: Tukey, Bonferroni and Scheffe, Statistica 6).

\section{Ancestral character state reconstruction}

Ancestral character state reconstruction analyses were carried out based on the phylogeny produced by the concatenation of genes from dataset (i). Both Maximum Parsimony and Maximum Likelihood approaches were tested using "Trace character History" option in Ancestral State Reconstruction package of MESQUITE 3.6 (Maddison \& Maddison 2018). As the number of states for the character "lifestyle" is large (10; Additional file 5: Table S5) a One-parameter Markov k-state model (Mk1; Lewis 2001) was used; it is a generalization of the JukesCantor model.

\section{RESULTS}

\section{Assembly statistics and completeness of the genomes}

After quality filtering, total numbers of PE reads for each species were 39.4 million for Lichenothelia convexa L1844, 9.7 million for L. intermixta L2282, 8.6 million for Saxomyces alpinus CCFEE 5470 and 9.9 million for S. americanus L1853. Assembly statistics are reported in Table 1. BUSCO assembly completeness analysis on 3156 orthologous genes for the subphylum Pezizomycotina recovered $93.1 \%$ for $L$. convexa, $92.3 \%$ for $L$. intermixta, $46.1 \%$ for S. alpinus and $95.7 \%$ for S. americanus. The mean and standard deviation for the entire assembly dataset of the 242 Dothideomycetes is $96.3 \pm 6$ (the complete output of the BUSCO analysis is reported in Additional file 6: Figure S1).

\section{Phylogenomic datasets}

The ">1Kb Gblocks" dataset comprises 242 samples (including the four newly sequenced species of Lichenothelia and Saxomyces) and 664 genes longer than $1000 \mathrm{bp}$ after Gblocks trimming. The total alignment length is $1.1 \mathrm{Mb}$. The "Complete Gblocks" dataset comprises the same samples, but it includes all the 2998 genes which are single copy, not predicted in multiple version and not empty after Gblocks trimming. As Gblocks does not allow gaps and it selects only perfectly aligned regions, many genes were drastically shortened. Hence the final length of the alignment is only twice as long as $(2.2 \mathrm{Mb})$ the "> $1 \mathrm{~Kb}$ Gblocks" dataset, which is a subset of the total. The presence of samples characterized by relevant events of gene duplication or low-quality assemblies (Additional file 6: Figure S1) hampered finding genes among BUSCO orthologs, which were in common among all samples. The number of samples was therefore reduced to 229 taxa in the "No missing" dataset, retaining all Lichenothelia and Saxomyces assemblies. A total of 63 genes and a $31 \mathrm{~Kb}$ alignment were used to run the phylogenetic inference. The complete " $>1 \mathrm{~Kb}$ GUIDANCE dataset” comprises all 242 samples and, as GUIDANCE tends to be less strict than Gblocks, 1260 genes longer than 1 $\mathrm{Kb}$ have been included in the final alignment with a length of 7.4 Mb (Additional file 9: Figure S4).

\section{Comparison of the inferred phylogenies}

Phylogenies inferred from the same dataset but applying different reconstruction methods showed highly similar topologies. Only three incongruences were detected when comparing the two phylogenies obtained from the concatenation ML inference and the coalescent-based species tree inference of the " $1 \mathrm{~Kb}$ Gblocks" dataset, namely the placements of Eremomyces bilateralis, Lineolata rhizophorae and Patellaria atrata (Fig. 2a, b). The RF distance between these two phylogenies is indeed only 0.109 , similar to the RF value obtained from the comparison of the two phylogenetic inferences based on the "1Kb GUIDANCE" dataset (0.100) (Additional file 2: Table S2 and Additional file 3: Table S3).

Changing both the starting dataset, ("Complete Gblocks" or "1Kb Gblocks") and the reconstruction method (concatenation or coalescent-based) generated the most diverse

Table 1 Summary information for sequencing and assembly of the genomes from four species in the genera Lichenothelia and Saxomyces

\begin{tabular}{lllllll}
\hline Sample & Reads (M) & Contings (> 500 bp) & Largest contig (bp) & N50 (bp) & L50 & Assembly size (Mbp) \\
\hline Lichenothelia convexa L1844 & 39.4 & 1669 & 539,045 & 61,89 & 165 & 36.6 \\
Lichenothelia intermixta L2282 & 9.7 & 3619 & 205,447 & 35,632 & 233 & 29.4 \\
Saxomyces alpinus CCFEE 5470 & 8.6 & 22,101 & 67,075 & 3086 & 3727 & 51.4 \\
Saxomyces americanus L1853 & 9.9 & 10,111 & 115,917 & 18,029 & 561 & 42.3
\end{tabular}

The states estimated to be the best according to the Likelihood threshold are marked by an asterisk (*) 


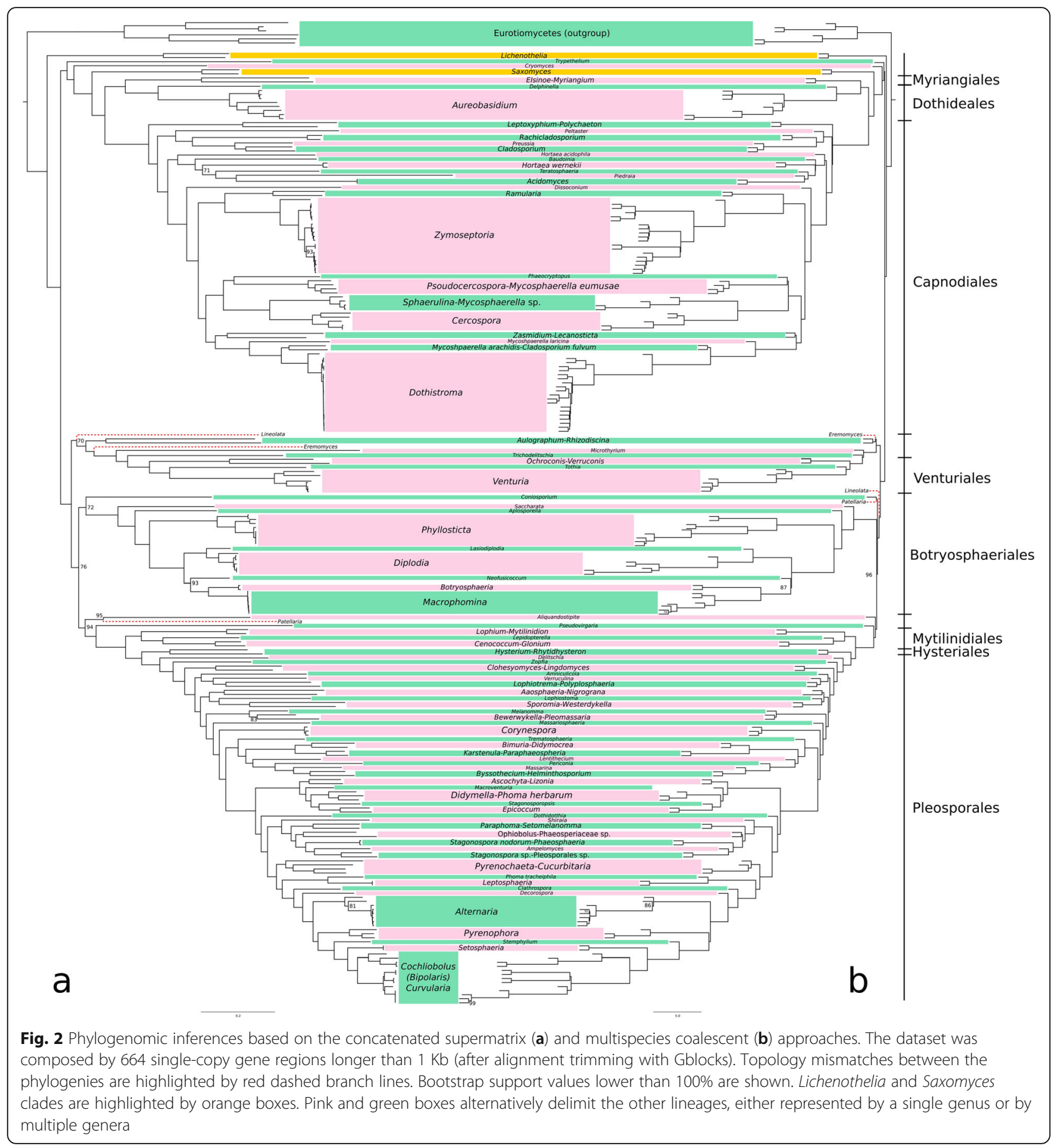

topologies (RF distance value of 0.117; Additional file 2: Table S2). The most similar topologies were produced by the coalescent-based approach on "1Kb Gblocks" and " $1 \mathrm{~Kb}$ GUIDANCE" dataset (Additional file 10: Figure S5). Although these inferences are based on a rather different datasets of genes, they produced nearly identical topologies with a RF distance of 0.025 (Additional file 2: Table S2). This is remarkable, because the datasets were comprised of 664 and 1260 genes, respectively. Even if the same markers were considered in both datasets, the retained parts of the alignment are not the same, as they have been obtained using two different filtering methods. Only Eremomyces bilateralis had a different placement, though unsupported (ultrafast bootstrap value lower than 95).

Three runs of the "1Kb Gblocks" dataset with the concatenation approach produced perfectly congruent topologies which only differs in the support values of some of the less-supported lineages, as shown by 
weighed RF distance values, which are very close to zero. Few other taxa show an unstable position within the phylogeny. Neofusicoccum parvum, for instance, is basal to the Botryosphaeria-Macrophomina clade using the " $1 \mathrm{~Kb}$ Gblocks" dataset, while it is basal to the LasidiplodiaDiplodia-Botryosphaeria-Macrophomina clade considering the "Complete Gblocks" dataset run as a concatenated supermatrix (Additional file 7: Figure S2). However, these phylogenetic positions are not fully supported by the ultrafast bootstrap value.

The "No missing" dataset, although built on both a reduced number of samples (229) and markers (63), produced highly similar results when considering the phylogeny obtained from the concatenation (Additional file 8: Figure S3a). However, when the same dataset is analyzed with the coalescent-based approach (Additional file 8: Figure S3b) the resulting RF distance between the two phylogenies is the highest recovered (0.181), though still rather low.

\section{Supermatrix resampling}

Phylogenetic analyses based on the randomly resampled, increasingly bigger alignment from " $1 \mathrm{~Kb}$ Gblocks" produced topologies which progressively approached the reference (Fig. 3). The results show both an increase of precision and accuracy when the sampling effort is increased. The increase in precision is highlighted by the RF distance among the same dimension resampled matrix topologies, becoming smaller as the amount of resampled columns is increased (Additional file 4: Table S4). The increase of accuracy is shown by the RF distance from the reference topology becoming progressively smaller (concatenation of "1Kb Gblocks" dataset; Fig. 3). The standard deviation (SD) also decreases from 0.026 (0.1\% resampling effort) to 0.011 (30\% resampling effort), highlighting a smaller distribution variance when the sampling effort is increased. ANOVA and post hoc tests show significant differences among increasing resampling effort up to $20 \%$ (ANOVA $p<0.01$; post hoc tests $\mathrm{p}<0.01$ ); conversely, increasing the resampling effort from 20 to $30 \%$ did not produce a significant shift of the distances from the reference topology.

\section{Ancestral character state reconstruction}

Maximum parsimony analysis highlighted the rockinhabiting lifestyle as the most parsimonious state for the basal node of the phylogeny (Table 2). The Maximum Likelihood approach estimated, instead, the saprotrophic lifestyle as the state with the best likelihood (-185.67), while the rock-inhabiting lifestyle was assigned the second best likelihood score (-186.41); all the other lifestyles are estimated with a remarkably lower likelihood value (Table 2).

\section{TAXONOMY}

Not applicable.

\section{DISCUSSION AND CONCLUSIONS}

The phylogenomic analyses presented here provide robust insight into evolutionary relationships within Dothideomycetes, with focus on the RIF genera Lichenothelia and Saxomyces. Exploring different combinations of markers, alignment filtering and phylogenetic reconstruction methods helped to find support among the overarching phylogenetic relationships. Our data suggest that the rock-inhabiting lifestyle is highly likely to be the ancestral state within this diverse class of fungi.

\section{Supermatrix resampling and phylogenomic inference}

Phylogenies are increasingly inferred from datasets comprising unprecedented numbers of samples and genetic markers. However, the number of samples and genetic loci, which both drive the accuracy of phylogenetic inferences, are often far from being comprehensive. Further, which of the two contributes more than the other in the phylogenetic accuracy remains debated, although empirical evidence tends to support the importance of extensive species sampling (Delsuc et al. 2005 and references therein).

Our taxon sampling of Dothideomycetes was determined by the availability of genomes, and we focused our attention on how the differences in the amount of information used for phylogenomic reconstructions may affect tree topology and nodal support. While the results of the resampling experiment should not be generalized, they provide insight into the robustness of phylogenomic inference. Even if samples with a high amount of missing data (up to $90 \%$ ) were included, we showed that a subset of the whole supermatrix, corresponding to about $20 \%$ of our dataset, provides a phylogenetic accuracy which did not improve significantly by further increasing the amount of data (Fig. 3). Moreover, the range of the RF distance of the $20 \%$ resampling effort distribution is even smaller than that registered when only the phylogenetic reconstruction method is changed on the complete supermatrix.

Strict alignment filtering criteria have been shown to worsen single-locus inference (Tan et al. 2015). However, in our analyses, strict filtering criteria did not significantly affect the resulting topologies when the length of each locus in the alignment was longer than $1 \mathrm{~kb}$. We adopted this threshold arbitrarily, without testing the effect of a progressively reduced length of each single marker on the resulting phylogenies. Though the effect of the alignment filtering was tested using a strict filtering strategy (Gblocks) and a relaxed strategy (GUIDANCE), we noticed that RF distances between phylogenies, whose 


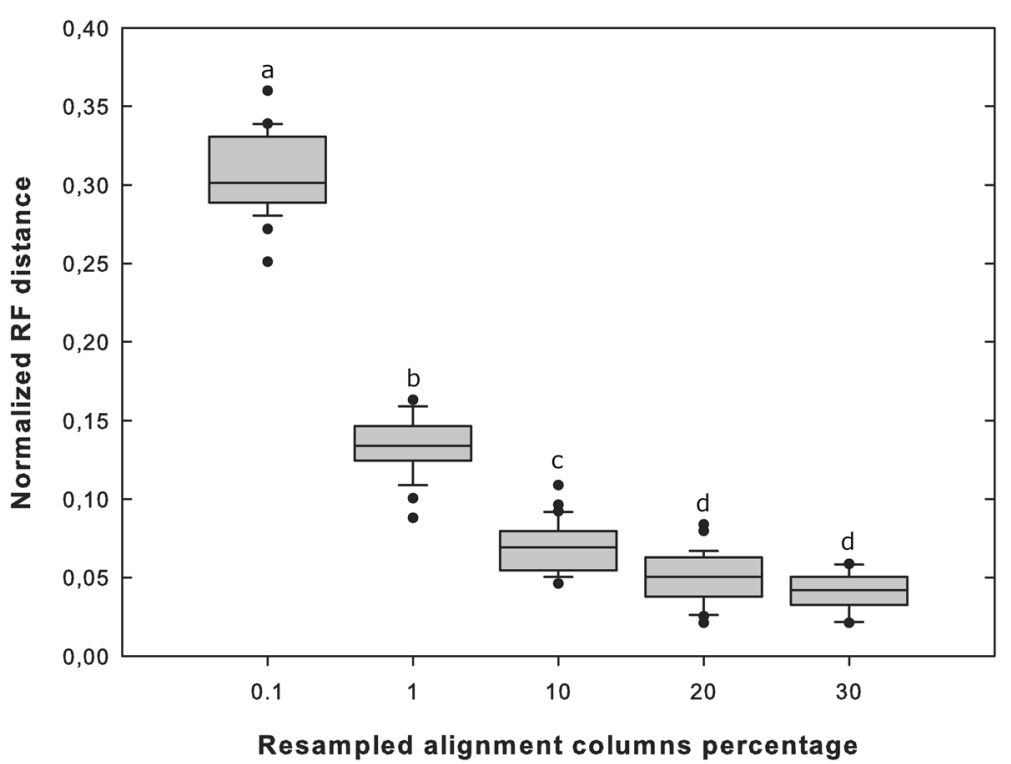

Fig. 3 Distribution of the 30 normalized RF distances from the reference topology for each of the five resampling efforts. Boxes are delimited by the distance between the 25th and 75th percentile; lines inside the boxes show the median value of the distribution; whiskers refer to 10th and 90th percentiles; outliers are marked with black dots. Letters $(\mathbf{a}, \mathbf{b}, \mathbf{c}, \mathbf{d})$ are used to label statistical significance; boxes with different letters are significantly different $(p<0.01)$ while the same letter indicates no statistical difference

pipelines only differ by the filtering step, are among the smallest registered (0.063 and 0.025; Additional file 2: Table S2). This highlights the stability of the phylogenetic signal in our genome-scale data in spite of differences in filtering strategies. In a similar way, even if diverse phylogenetic reconstruction methods were implemented, they produced consistent phylogenies with low RF values, supporting the utility of concatenation-based phylogenomic inference methods,

Table 2 Ancestral character state reconstruction Log Likelihood values for the basal node of the ">1 Kb Gblocks" dataset phylogeny. The states estimated to be the best according to the Likelihood threshold are marked with an asterisk. LIC: lichen; RIF: rock-inhabiting fungus; PP: plant pathogen; SAP: saprotroph; EPI: epiphyte; AP: animal pathogen; FP: fungi pathogen; ECT: ectomycorrhiza; END: endophyte; EXT: extremophile

\begin{tabular}{ll}
\hline Lifestyle & Log Likelihood \\
\hline LIC & -190.27 \\
RIF & $-186.41^{*}$ \\
PP & -189.95 \\
SAP & $-185.67^{*}$ \\
EPI & -190.50 \\
AP & -190.51 \\
FP & -190.53 \\
ECT & -190.53 \\
END & -190.53 \\
EXT & -190.51 \\
\hline
\end{tabular}

in particular relating to taxon sampling and lineage sorting conditions of our dataset.

Concatenation approaches have been shown to produce highly supported but wrong topologies when sufficiently short branch lengths are generated in relation to effective population size (Kubatko and Degnan 2007). In the present study, coalescent-based approaches were not applied on the whole set of markers because single-locus inferences were heavily affected either by difficult-toalign regions or by strict filtering criteria. The latter would highly reduce the marker length and consequently their phylogenetic signal, leading to single-locus phylogenies dominated by stochastic error (Jeffroy et al. 2006). Here, the coalescent-based method ASTRAL III was applied using 664 individual BUSCO gene topologies (Fig. 2b), resulting in relationships that were largely consistent with the concatenated supermatrix approaches (Fig. 2a). Future, coalescent-based inferences in Dothideomycetes would benefit from a more extensive taxon sampling - a condition not met for many of the species represented by genomic data in this study. Large-scale sequencing projects, such as the "One thousand fungal genomes" (http://1000.fungalgenomes.org) and other laboratories which investigate hidden fungal biodiversity, are filling this gap, sequencing fungal genomes from the least known families. This effort, from a phylogenetic point of view, will make the taxon sampling more comprehensive and, therefore, the phylogenetic inference in Dothideomycetes more accurate. 
As we did not find significant topological discrepancies among methods with varying degrees of sensitivity to the noise of phylogenetic signal caused by sequence substitution saturation and/or compositional bias, we refrained from testing targeted filtering approaches (e.g. the exclusion of variable third-codon positions from the alignment) or the use of amino acid alignments instead of nucleotide sequences. The latter would exploit the redundancy of the genetic code to better "preserve" phylogenetic signal (Jeffroy et al. 2006). However, these possible sources of noise should be considered when the most ancient phylogenetic relationships of the tree of life are investigated.

A dataset without any missing data was also tested. In this case, the phylogenetic inferences were reconstructed only based on 63 markers common to all the samples and when certain samples containing high amount of missing data were excluded (e.g. Rachicladosporium due to massive gene duplication). We anticipated that this drastic reduction of genetic markers would likely have a greater effect on the resulting phylogeny than the missing data. However, we recovered largely the same topology with the dataset of 664 concatenated genes. Only minor differences concerning the placement of single samples were noted, but these did not involve the most basal nodes. In accordance with other studies which tested real and simulated data (Driskell et al. 2004; Philippe et al. 2004), the phylogenomic dataset assembled here is not negatively affected by missing data, as the incomplete sequences are still represented by enough informative characters.

\section{Comparison to published Dothideomycetes phylogenies and the phylogenetic placement of RIF}

Phylogenomic data have contributed to a shift in our view of evolution, reshuffling many deep evolutionary relationships along the tree of life (Rokas et al. 2003; Fitzpatrick et al. 2006; Wang et al. 2009). In addition, the increasing availability of fungal genomes allows us to investigate evolutionary histories at a finer taxonomic scale. A consistent comparison of tree topologies among previously published phylogenies and the genome-based phylogeny inferred in this study is still not straightforward, because many orders reported in other studies are missing in our phylogenomic inference. Nevertheless, it was possible to detect the main clades which were identified previously and whose phylogenetic position were confirmed by our inference. In general, Dothideomycetes phylogenies published to date identified well-defined lineages at both order and family levels; however, some relationships have remained unsettled. Genome-based inference, as used here, helped to clarify these relationships but cannot be considered the final step to explain all the evolutionary relationships, as limitations concerning the phylogenetic signal of the data and limits of the reconstruction methods can still affect the results. Moreover, sequence availability is still the most relevant bottleneck in phylogenomics, though the scenario is changing quickly.

The subclasses Dothideomycetidae, which includes the orders Capnodiales, Myriangiales and Dothideales, and Pleosporomycetidae, which includes the orders Pleosporales, Mytilinidiales and Hysteriales, have been recovered as highly supported lineages in previous traditional multilocus approaches (Ruibal et al. 2009; Schoch et al. 2009; Muggia et al. 2015; Liu et al. 2017; Ametrano et al. 2019) as well as in the present analyses. Hysteriales and Pleosporales have been recurrently identified as sister clades within Pleosporomycetidae, with the lineage Mytilinidiales basal to them; only Hyde et al. (2013) reported Hysteriales as sister to Mytilinidiales. The relationships of taxa within the subclass Dothideomycetidae is congruent among all the other previous phylogenies and in the present one, confirming Myriangiales and Dothideales as sister groups and Capnodiales as basal to them. The placement of Botryosphaeriales agrees with previous phylogenies (e.g. Ruibal et al. 2009), being basal to the orders belonging to the subclass Pleosporomycetidae. In contrast, the phylogenetic position of Venturiales differs substantially in the present study from other recent multilocus phylogenies produced by Hyde et al. (2013) and Liu et al. (2017). These inferences, indeed, place Venturiales as related to the subclass Dothideomycetidae (e.g. orders Dothideales, Myriangiales and Capnodiales), while our analyses recovered it at the base of the subclass Pleosporomycetidae and order Botryosphaeriales, whereas its relationship with Microthyriales is maintained.

Lichenothelia and Saxomyces are confirmed to be distinct, independent lineages, as recently reported by Ametrano et al. (2019). However, their placement within Dothideomycetes differs when the inference is based on genomic data. Here Lichenothelia samples were recovered as basal to the Dothideomycetes, while Saxomyces is early diverging in Dothideomycetidae, diverging from the rest of the clade after Trypethelium and Cryomyces. The placement of the other two genera of extremotolerant black fungi Rachicladosporium and Hortaea is congruently found here within Capnodiales, as in previous multilocus phylogenies (Crous et al. 2009). The placement of Lichenothelia and Saxomyces is particularly interesting because it highlights once more the connection between the lichenized and the not-lichenized lifestyles intrinsic within the two genera (Hawksworth 1981; Muggia et al. 2013, 2015; Ametrano et al. 2017, 2019). It also further strengthens the hypothesis that the earliest diverging taxa of the subclass Dothideomycetidae were rock inhabitants that might have been able to form a lichen-like association with algae. This is in accordance 
with previous studies where the rock-inhabiting lifestyle was suggested as ancestral for Dothideomycetes and Chaetothyriomycetes (Gueidan et al. 2008). This hypothesis is also supported for Dothideomycetes by our analyses, as both Maximum Parsimony and Maximum Likelihood ancestral character state reconstructions estimate the highest and the second highest score for the rock-inhabiting lifestyle, respectively. The results highlight this lifestyle as ancestral, though taxon sampling within the phylogeny is heavily biased by the lifestyles of those taxa which have been preferentially sequenced due to their economical and biotechnological potential, such as saprotroph and plant pathogens. In the superclass Dothideomyceta, rock inhabitants are also known from Capnodiales (Egidi et al. 2014), of which Rachicladosporium is a representative in this study, and Lichenostigmatales (Ertz et al. 2014).

Future studies, investigating character evolution on a more comprehensive genome dataset, representing a more equilibrate proportion of lifestyles within the class, will elucidate the evolution of the different lifestyles in this dynamic fungal class.

\section{Supplementary information}

Supplementary information accompanies this paper at https://doi.org/10. 1186/s43008-019-0018-2.

Additional file 1: Table S1. Assemblies retrieved from the NCBI and JGI genome portals and included in the phylogenomic analyses.

Additional file 2: Table S2. RF distances and normalized RF distance among the main phylogenies generated from the datasets containing the complete set of samples. Three distances are reported: a regular RF distance and two flavours of weighted RF distance as reported in the RAxML 8.2 manual. Numbers in the first two columns refer to the following starting datasets and reconstruction methods: (0) "> $1 \mathrm{~kb}$ Gblocks" IQTree run 1; (1) "> 1 kb Gblocks" IQTree run 2; (2) "> 1 kb Gblocks" IQTree run 3; (3) "> 1 kb Gblocks" ASTRAL; (4) "Complete Gblocks" IQTree; (5) ">1Kb GUIDANCE" IQTree; (6) ">1Kb GUIDANCE" ASTRAL.

Additional file 3: Table S3. RF distances and normalized RF distances from the reference topology of the 30 resampled matrices for each resampling effort value $(0.1-30 \%)$.

Additional file 4: Table S4. Average values and standard deviation (SD) of RF distances and normalized RF distances among the 30 phylogenies generated from the randomly resampled matrix with the same resampling effort.

Additional file 5: Table S5. Taxa and associated lifestyle used in ancestral character state reconstruction (lichen: 0 ; rock-inhabiting: 1; plant pathogen: 2; saprotroph: 3; epiphyte: 4; animal pathogen: 5; fungi pathogen: 6; ectomycorrhiza: 7; endophyte: 8; extremophile: 9).

Additional file 6: Figure S1. Assembly completeness on the base of 3156 Pezizomycotina orthologs evaluated by BUSCO and expressed as the percentage of complete (green), duplicated (pink) and fragmented or missing (grey) genes. Distribution outliers are highlighted with "**".

Additional file 7: Figure S2. Phylogeny generated from 2998 concatenated genes of the "Complete Gblocks" dataset with IQTree.

Additional file 8: Figure S3. Phylogenies generated from 63 genes of the "No missing" dataset; (a) ML tree generated with IQTree on concatenated genes; (b) ASTRAL III species tree.
Additional file 9: Figure S4. Phylogeny generated from 1260 concatenated genes of the ">1 Kb GUIDANCE" dataset with IQTree.

Additional file 10: Figure S5. Phylogeny generated from 1260 concatenated genes of the ">1Kb GUIDANCE" dataset with ASTRAL.

\section{Abbreviations}

ASTRAL: Accurate Species TRee Algorithm; BUSCO: Benchmarking Universal Single Copy Orthologs; CCFEE: Culture Collection of Fungi from Extreme Environments; GUIDANCE: GUIDe tree-based AligNment ConfidencE; JGI: Joint Genome Institute; MAFFT: Multiple Alignment using Fast Fourier Transform; NCBI: National Center for Biotechnology Information; PE: Pairedend; RAxML: Randomized Accelerated Maximum Likelihood; RF: RobinsonFoulds; RIF: Rock inhabiting fungi

\section{Acknowledgments}

We thank Joseph Spatafora, Patrik Inderbitzin, Gerald F. Bills and David Ezra to allow the use of the unpublished genome assemblies. The Italian Antarctic National Museum (MNA) is acknowledged for financial support for the CCFEE (Culture Collection of Fungi from Extreme Environments) stored in the Mycological Section on the MNA, University of Tuscia, Viterbo (I).

Adherence to national and international regulations Not applicable

\section{Authors' contributions}

CGA conceived the study, performed wet lab procedures, data collection, data analyses and major contribution in writing the manuscript; FG performed data analyses and manuscript drafting; PWC, SBG and CL produced part of the available, still unpublished genome assemblies and manuscript drafting; LS performed Saxomyces alpinus culture isolation and manuscript drafting; HTL and STL performed manuscript drafting; LM conceived the study, performed cultures isolation and major contribution in writing the manuscript. All authors read and approved the final manuscript.

\section{Funding}

The research was funded by the project Finanziamenti di Ateneo per progetti di Ricerca scientifica (FRA2014) of the University of Trieste assigned to $L M$, by The Grainger Bioinformatics Center and the Negaunee Foundation, and the National natural science foundation of China (No. 30870010).

\section{Availability of data and materials}

Alignments used in this study are available at https://zenodo.org/record/343 0636\#.XYDFOfexVY8. Resampling as well as alignment filtering by length or gap percentage were performed using Python3 scripts (available in GitHub: https://github.com/clof84/Alignment_filtering_and_resampling).

Genome assemblies used in this study are available in GenBank and/or JGl Genome portal.

Ethics approval and consent to participate

Not applicable

\section{Consent for publication}

Permission to use unpublished genome assembly have been asked to the owners.

Competing interests

The authors declare that they have no competing interests.

\section{Author details}

${ }^{1}$ Department of Life Sciences, University of Trieste, via Giorgieri 10, 34127 Trieste, Italy. ${ }^{2}$ Grainger Bioinformatics Center and Integrative Research Center, Science and Education, Field Museum of Natural History, 1400 S Lake Shore Drive, Chicago, IL 60605, USA. ${ }^{3}$ Westerdijk Fungal Biodiversity Institute, P.O. Box 85176, 3508, AD, Utrecht, The Netherlands. ${ }^{4}$ USDA-ARS, Crop Production and Pest Control Research Unit and Department of Botany and Plant Pathology, Purdue University, 915 West State Street, West Lafayette, IN 47907-2054, USA. ${ }^{5}$ College of Plant Health and Medicine, Qingdao Agricultural University, Qingdao 266109, China. ${ }^{6}$ Department of Ecological and Biological Sciences, University of Tuscia, Largo dell' Università, 01100 Viterbo, Italy. ${ }^{7}$ Italian National Antarctic Museum (MNA), Mycological Section, 
Genoa, Italy. ${ }^{8}$ Department of Biology and M.L. Bean Life Science Museum, Brigham Young University, 4102 Life Science Building, Provo, UT 84602, USA.

\section{Received: 24 June 2019 Accepted: 11 September 2019}

Published online: 30 October 2019

\section{References}

Ahmadjian V (1967) The lichen symbiosis. Blaisdell Publishing Co. 250 p, Massachusetts

Ametrano CG, Knudsen K, Kocourková J, Grube M, Selbmann L, Muggia L (2019) Phylogenetic relationships of rock-inhabiting black fungi belonging to the widespread genera Lichenothelia and Saxomyces. Mycologia 111(1):127-160

Ametrano CG, Selbmann L, Muggia L (2017) A standardized approach for coculturing dothidealean rock-inhabiting fungi and lichen photobionts in vitro. Symbiosis 73(1):35-44

Aragona M, Minio A, Ferrarini A et al (2014) De novo genome assembly of the soil-borne fungus and tomato pathogen Pyrenochaeta lycopersici. BMC Genomics 15(1):313

Bankevich A, Nurk S, Antipov D et al (2012) SPAdes: a new genome assembly algorithm and its applications to single-cell sequencing. Journal of Computational Biology 19(5):455-477

Bihon W, Wingfield MJ, Slippers B, Duong TA, Wingfield BD (2014) MAT gene idiomorphs suggest a heterothallic sexual cycle in a predominantly asexual and important pine pathogen. Fungal Genetics and Biology 62:55-61

Blanco-Ulate B, Rolshausen P, Cantu D (2013) Draft genome sequence of Neofusicoccum parvum isolate UCR-NP2, a fungal vascular pathogen associated with grapevine cankers. Genome Announcements 1(3): e00339-e00313

Bock CH, Chen C, Yu F, Stevenson KL, Wood BW (2016) Draft genome sequence of Fusicladium effusum, cause of pecan scab. Standards in Genomic Sciences 11(1):36

Bolger AM, Lohse M, Usadel B (2014) Trimmomatic: a flexible trimmer for Illumina sequence data. Bioinformatics 30(15):2114-2120

Castresana J (2000) Selection of conserved blocks from multiple alignments for their use in phylogenetic analysis. Molecular Biology and Evolution 17(4): $540-552$

Chan CL, Yew SM, Na SL et al (2014) Draft genome sequence of Ochroconis constricta UM 578, isolated from human skin scraping. Genome Announcements 2(2):e00074-e00014

Chan CX, Ragan MA (2013) Next-generation phylogenomics. Biology Direct 8(1):3

Chan GF, Bamadhaj HM, Gan HM, Rashid NAA (2012) Genome sequence of Aureobasidium pullulans AY4, an emerging opportunistic fungal pathogen with diverse biotechnological potential. Eukaryotic Cell 11(11):1419-1420

Chang TC, Salvucci A, Crous PW, Stergiopoulos I (2016) Comparative genomics of the Sigatoka disease complex on banana suggests a link between parallel evolutionary changes in Pseudocercospora fijiensis and Pseudocercospora eumusae and increased virulence on the banana host. PLoS Genetics 12(8):e1005904

Coleine C, Masonjones S, Selbmann L, Zucconi L, Onofri S, Pacelli C, Stajich JE (2017) Draft genome sequences of the Antarctic endolithic fungi Rachicladosporium antarcticum CCFEE 5527 and Rachicladosporium sp. CCFEE 5018. Genome Announcements 5(27):e00397-e00317

Condon BJ, Leng Y, Wu D et al (2013) Comparative genome structure, secondary metabolite, and effector coding capacity across Cochliobolus pathogens. PLoS Genetics 9(1):e1003233

Cooke IR, Jones D, Bowen JK et al (2014) Proteogenomic analysis of the Venturia pirina (pear scab fungus) secretome reveals potential effectors. Journal of Proteome Research 13(8):3635-3644

Crous PW, Schoch CL, Hyde KD, Wood AR, Gueidan C, De Hoog GS, Groenewald JZ (2009) Phylogenetic lineages in the Capnodiales. Studies in Mycology 64:17-47

Delsuc F, Brinkmann H, Philippe H (2005) Phylogenomics and the reconstruction of the tree of life. Nature Reviews. Genetics 6(5):361

Desjardins CA, Champion MD, Holder JW et al (2011) Comparative genomic analysis of human fungal pathogens causing paracoccidioidomycosis. PLoS Genetics 7(10):e1002345

Driskell AC, Ané C, Burleigh JG, McMahon MM, O'Meara BC, Sanderson MJ (2004) Prospects for building the tree of life from large sequence databases. Science 306(5699):1172-1174
Egidi E, De Hoog GS, Isola D et al (2014) Phylogeny and taxonomy of meristematic rock-inhabiting black fungi in the Dothideomycetes based on multi-locus phylogenies. Fungal Diversity 65(1):127-165

Ellwood SR, Liu Z, Syme RA et al (2010) A first genome assembly of the barley fungal pathogen Pyrenophora teres f. teres. Genome Biology 11(11):R109

Ertz D, Lawrey JD, Common RS, Diederich P (2014) Molecular data resolve a new order of Arthoniomycetes sister to the primarily lichenized Arthoniales and composed of black yeasts, lichenicolous and rock-inhabiting species. Fungal Diversity 66(1):113-137

Fedorova ND, Khaldi N, Joardar VS et al (2008) Genomic islands in the pathogenic filamentous fungus Aspergillus fumigatus. PLoS Genetics 4(4):e1000046

Franco ME, López S, Medina R, Saparrat MC, Balatti P (2015) Draft genome sequence and gene annotation of Stemphylium lycopersici strain CIDEFI-216. Genome Announcements 3(5):e01069-e01015

Fitzpatrick DA, Logue ME, Stajich JE, Butler G (2006) A fungal phylogeny based on 42 complete genomes derived from supertree and combined gene analysis. BMC Evolutionary Biology 6:99

Galagan JE, Calvo SE, Cuomo C et al (2005) Sequencing of Aspergillus nidulans and comparative analysis with A. fumigatus and A. oryzae. Nature 438(7071):1105

Gao S, Li Y, Gao J, Suo Y, Fu K, Li Y, Chen J (2014) Genome sequence and virulence variation-related transcriptome profiles of Curvularia lunata, an important maize pathogenic fungus. BMC Genomics 15(1):627

Goodwin SB, M'barek SB, Dhillon B et al (2011) Finished genome of the fungal wheat pathogen Mycosphaerella graminicola reveals dispensome structure, chromosome plasticity, and stealth pathogenesis. PLOS Genetics 7(6):e1002070

Gostinčar C, Lenassi M, Gunde-Cimerman N, Plemenitaš A (2011) Fungal adaptation to extremely high salt concentrations. In: Advances in Applied Microbiology, vol 77, pp 71-96

Gostinčar C, Muggia L, Grube M (2012) Polyextremotolerant black fungi: oligotrophism, adaptive potential, and a link to lichen symbioses. Frontiers in Microbiology 3:90

Gostinčar C, Ohm RA, Kogej T et al (2014) Genome sequencing of four Aureobasidium pullulans varieties: biotechnological potential, stress tolerance, and description of new species. BMC Genomics 15(1):549

Grandaubert J, Bhattacharyya A, Stukenbrock EH (2015) RNA-seq based gene annotation and comparative genomics of four fungal grass pathogens in the genus Zymoseptoria identify novel orphan genes and species-specific invasions of transposable elements. G3: Genes, Genomes, Genetics 5: 13231333

Gueidan C, Roux C, Lutzoni F (2008) A rock-inhabiting ancestor for mutualistic and pathogen-rich fungal lineages. Studies in Mycology 61:111-119

Gurevich A, Saveliev V, Vyahhi N, Tesler G (2013) QUAST: quality assessment tool for genome assemblies. Bioinformatics 29(8):1072-1075

Han WB, Lu YH, Zhang AH et al (2014) Curvulamine, a new antibacterial alkaloid incorporating two undescribed units from a Curvularia species. Organic Letters 16(20):5366-5369

Hane JK, Lowe RG, Solomon PS (2007) Dothideomycete-plant interactions illuminated by genome sequencing and EST analysis of the wheat pathogen Stagonospora nodorum. Plant Cell 19(11):3347-3368

Hawksworth DL (1981) Lichenothelia, a new genus for the Microthelia aterrima group. The Lichenologist 13(2):141-153

Hoang DT, Chernomor O, von Haeseler A, Minh BQ, Vinh LS (2017) UFBoot2: improving the ultrafast bootstrap approximation. Molecular Biology and Evolution 35(2):518-522

Hu J, Chen C, Peever T, Dang H, Lawrence C, Mitchell T (2012) Genomic characterization of the conditionally dispensable chromosome in Alternaria arborescens provides evidence for horizontal gene transfer. BMC Genomics 13(1):171

Hyde KD, Jones EG, Liu JK et al (2013) Families of Dothideomycetes. Fungal Diversity 63(1):1-313

Jaklitsch W, Baral HO, Lücking R, Lumbsch HT, Frey W (2016) Syllabus of plant families-a. Engler's syllabus der Pflanzenfamilien part 1/2

Jeffroy O, Brinkmann H, Delsuc F, Philippe H (2006) Phylogenomics: the beginning of incongruence? Trends in Genetics 22(4):225-231

Joardar V, Abrams NF, Hostetler J et al (2012) Sequencing of mitochondrial genomes of nine Aspergillus and Penicillium species identifies mobile introns and accessory genes as main sources of genome size variability. BMC Genomics 13(1):698 
Katoh K, Standley DM (2013) MAFFT multiple sequence alignment software version 7: improvements in performance and usability. Molecular Biology and Evolution 30(4):772-780

Knapp DG, Németh JB, Barry K et al (2018) Comparative genomics provides insights into the lifestyle and reveals functional heterogeneity of dark septate endophytic fungi. Scientific Reports 8:6321

Kuan CS, Yew SM, Toh YF et al (2015) Dissecting the fungal biology of Bipolaris papendorfii: from phylogenetic to comparative genomic analysis. DNA Research 22:219-232

Kubatko LS, Degnan JH (2007) Inconsistency of phylogenetic estimates from concatenated data under coalescence. Systematic Biology 56(1):17-24

Kück P, Meusemann K (2010) FASconCAT: convenient handling of data matrices. Molecular Phylogenetics and Evolution 56(3):1115-1118

Lenassi M, Gostinčar C, Jackman S et al (2013) Whole genome duplication and enrichment of metal cation transporters revealed by de novo genome sequencing of extremely halotolerant black yeast Hortaea werneckii. PLoS One 8(8):e71328

Lewis PO (2001) Phylogenetic systematics turns over new leaf. Trends in Ecology \& Evolution 16:30-37

Liu JK, Hyde KD, Jeewon R et al (2017) Ranking higher taxa using divergence times: a case study in Dothideomycetes. Fungal Diversity 84(1):75-99

Lopez D, Ribeiro S, Label P et al (2018) Genome-wide analysis of Corynespora cassiicola leaf fall disease putative effectors. Frontiers in Microbiology 9:276

Maddison WP, Maddison DR (2018) Mesquite: a modular system for evolutionary analysis Version 3.6 http://www.mesquiteproject.org

Marsberg A, Kemler M, Jami F et al (2017) Botryosphaeria dothidea: a latent pathogen of global importance to woody plant health. Molecular Plant Pathology 18(4):477-488

Mondo SJ, Dannebaum RO, Kuo RC et al (2017) Widespread adenine N6methylation of active genes in fungi. Nature Genetics 49(6):964

Morales-Cruz A, Amrine KC, Blanco-Ulate B et al (2015) Distinctive expansion of gene families associated with plant cell wall degradation, secondary metabolism, and nutrient uptake in the genomes of grapevine trunk pathogens. BMC Genomics 16(1):469

Mosier AC, Miller CS, Frischkorn KR et al (2016) Fungi contribute critical but spatially varying roles in nitrogen and carbon cycling in acid mine drainage. Frontiers in Microbiology 7:238

Muggia L, Gueidan C, Knudsen K, Perlmutter G, Grube M (2013) The lichen connections of black fungi. Mycopathologia 175:523-535

Muggia L, Kocourkova J, Knudsen K (2015) Disentangling the complex of Lichenothelia species from rock communities in the desert. Mycologia 107:1233-1253

Nai C, Wong HY, Pannenbecker A, Broughton WJ, Benoit I, de Vries RP, Gueidan C, Gorbushina AA (2013) Nutritional physiology of a rock-inhabiting, mode microcolonial fungus from an ancestral lineage of the Chaetothyriales (ascomycetes). Fungal Genetics and Biology 56:54-66

Nelsen MP, Lücking R, Grube M, Mbatchou JS, Muggia L, Plata ER, Lumbsch HT (2009) Unravelling the phylogenetic relationships of lichenised fungi in Dothideomyceta. Studies in Mycology 64:135-144

$\mathrm{Ng}$ KP, Yew SM, Chan CL et al (2012) Sequencing of Cladosporium sphaerospermum, a dematiaceous fungus isolated from blood culture. Eukaryotic Cell 11(5):705-706

Nguyen HD, Lewis CT, Lévesque CA, Gräfenhan T (2016) Draft genome sequence of Alternaria alternata ATCC 34957. Genome Announcements 4(1):e01554-e01515

Nguyen LT, Schmidt HA, von Haeseler A, Minh BQ (2014) IQ-TREE: a fast and effective stochastic algorithm for estimating maximum-likelihood phylogenies. Molecular Biology and Evolution 32(1):268-274

Ohm RA, Feau N, Henrissat B et al (2012) Diverse lifestyles and strategies of plant pathogenesis encoded in the genomes of eighteen Dothideomycetes fungi. PLoS Pathogens 8(12):e1003037

Orner VA, Cantonwine EG, Wang XM et al (2015) Draft genome sequence of Cercospora arachidicola, causal agent of early leaf spot in peanuts. Genome Announcements 3(6):e01281-e01215

Penn O, Privman E, Landan G, Graur D, Pupko T (2010) An alignment confidence score capturing robustness to guide-tree uncertainty. Molecular Biology and Evolution 27(8):1759-1767

Peter M, Kohler A, Ohm RA, Kuo A, Krützmann J, Morin E, Clum A (2016) Ectomycorrhizal ecology is imprinted in the genome of the dominant symbiotic fungus Cenococcum geophilum. Nature Communications 7: 12662
Philippe H, Snell EA, Bapteste E, Lopez P, Holland PW, Casane D (2004) Phylogenomics of eukaryotes: impact of missing data on large alignments. Molecular Biology and Evolution 21(9):1740-1752

Prenafeta-Boldu FX, Summerbell R, de Hoog SG (2006) Fungi growing on aromatic hydrocarbons: biotechnology's unexpected encounter with biohazard? FEMS Microbiology Reviews 30:109-130

Raffaele S, Kamoun S (2012) Genome evolution in filamentous plant pathogens: why bigger can be better. Nature Reviews. Microbiology 10(6):417-430

Robinson DF, Foulds LR (1981) Comparison of phylogenetic trees. Mathematical Biosciences 53(1-2):131-147

Rokas A, Williams BL, King N, Carroll SB (2003) Genome-scale approaches to resolving incongruence in molecular phylogenies. Nature 425(6960):798

Rouxel T, Grandaubert J, Hane JK et al (2011) Effector diversification within compartments of the Leptosphaeria maculans genome affected by repeatinduced point mutations. Nature Communications 2:202

Ruibal C, Gueidan C, Selbmann L et al (2009) Phylogeny of rock-inhabiting fungi related to Dothideomycetes. Studies in Mycology 64:123-133

Schoch CL, Crous PW, Groenewald JZ et al (2009) A class-wide phylogenetic assessment of Dothideomycetes. Studies in Mycology 64:1-15

Schoch CL, Shoemaker RA, Seifert KA, Hambleton S, Spatafora JW, Crous PW (2006) A multigene phylogeny of the Dothideomycetes using four nuclear loci. Mycologia 98(6):1041-1052

Selbmann L, Isola D, Egidi E, Zucconi L, Gueidan C, de Hoog GS, Onofri S (2014) Mountain tips as reservoirs for new rock-fungal entities: Saxomyces gen. Nov. and four new species from the alps. Fungal Diversity 65:167-182

Sharpton TJ, Stajich JE, Rounsley SD et al (2009) Comparative genomic analyses of the human fungal pathogens Coccidioides and their relatives. Genome Research 19(10):1722-1731

Shaw JJ, Spakowicz DJ, Dalal RS et al (2015) Biosynthesis and genomic analysis of medium-chain hydrocarbon production by the endophytic fungal isolate Nigrograna mackinnonii E5202H. Applied Microbiology and Biotechnology 99(8):3715-3728

Shiller J, Van de Wouw AP, Taranto AP et al (2015) A large family of AvrLm6-like genes in the apple and pear scab pathogens, Venturia inaequalis and Venturia pirina. Frontiers in Plant Science 6:980

Shrestha SK, Cochran A, Mengistu A, Lamour K, Castro-Rocha A, Young-Kelly H (2017) Genetic diversity, Qol fungicide resistance, and mating type distribution of Cercospora sojina-implications for the disease dynamics of frogeye leaf spot on soybean. PLoS One 12(5):e0177220

Soliai MM, Meyer SE, Udall JA et al (2014) De novo genome assembly of the fungal plant pathogen Pyrenophora semeniperda. PLoS One 9(1):e87045

Spatafora JW, Owensby CA, Douhan GW, Boehm EW, Schoch CL (2012) Phylogenetic placement of the ectomycorrhizal genus Cenococcum in Gloniaceae (Dothideomycetes). Mycologia 104(3):758-765

Stamatakis A (2014) RAxML version 8: a tool for phylogenetic analysis and postanalysis of large phylogenies. Bioinformatics 30(9):1312-1313

Sterflinger K (2006) Black yeasts and meristematic fungi: ecology, diversity and identification. In: Rosa CA, Peter G (eds) Biodiversity and ecophysiology of yeasts. Springer, Berlin Heidelberg, pp 501-514

Sterflinger K, Lopandic K, Pandey RV, Blasi B, Kriegner A (2014) Nothing special in the specialist? Draft genome sequence of Cryomyces antarcticus, the most extremophilic fungus from Antarctica. PLoS One 9(10):e109908

Stukenbrock EH, Bataillon T, Dutheil JY et al (2011) The making of a new pathogen: insights from comparative population genomics of the domesticated wheat pathogen Mycosphaerella graminicola and its wild sister species. Genome Research 21(12):2157-2166

Tan G, Muffato M, Ledergerber C, Herrero J, Goldman N, Gil M, Dessimoz C (2015) Current methods for automated filtering of multiple sequence alignments frequently worsen single-gene phylogenetic inference. Systematic Biology 64(5):778-791

Teixeira MDM, Moreno LF, Stielow BJ et al (2017) Exploring the genomic diversity of black yeasts and relatives (Chaetothyriales, Ascomycota). Studies in Mycology 86:1-28

Thompson WR (1935) On the criterion for the rejection of observations and the distribution of the ratio of deviation to sample standard deviation. Annals of Mathematical Statistics 6(4):214-219

Vaghefi N, Ades PK, Hay FS, Pethybridge SJ, Ford R, Taylor PW (2015) Identification of the MAT1 locus in Stagonosporopsis tanaceti, and exploring its potential for sexual reproduction in Australian pyrethrum fields. Fungal Biology 119(5):408-419 
Van der Nest MA, Bihon W, De Vos L et al (2014) Draft genome sequences of Diplodia sapinea, Ceratocystis manginecans, and Ceratocystis moniliformis. IMA Fungus 5(1):135-140

Verma S, Gazara RK, Nizam S, Parween S, Chattopadhyay D, Verma PK (2016) Draft genome sequencing and secretome analysis of fungal phytopathogen Ascochyta rabiei provides insight into the necrotrophic effector repertoire. Scientific Reports 6:24638

Wang H, Xu Z, Gao L, Hao B (2009) A fungal phylogeny based on 82 complete genomes using the composition vector method. BMC Evolutionary Biology 9(1):195

Wang M, Sun X, Yu D, Xu J, Chung K, Li H (2016) Genomic and transcriptomic analyses of the tangerine pathotype of Alternaria alternata in response to oxidative stress. Scientific Reports 6:32437

Waterhouse RM, Seppey M, Simão FA, Manni M, loannidis $P$, Klioutchnikov G, Zdobnov EM (2017) BUSCO applications from quality assessments to gene prediction and phylogenomics. Molecular Biology and Evolution 35(3):543-548

Wingfield BD, Ades PK, Al-Naemi FA et al (2015) IMA genome-F 4: draft genome sequences of Chrysoporthe austroafricana, Diplodia scrobiculata, Fusarium nygamai, Leptographium lundbergii, Limonomyces culmigenus, Stagonosporopsis tanaceti, and Thielaviopsis punctulata. IMA fungus 6(1):233-248

Xu C, Chen H, Gleason ML, Xu JR, Liu H, Zhang R, Sun G (2016) Peltaster fructicola genome reveals evolution from an invasive phytopathogen to an ectophytic parasite. Scientific Reports 6:22926

Yang H, Wang Y, Zhang Z, Yan R, Zhu D (2014) Whole-genome shotgun assembly and analysis of the genome of Shiraia sp. strain Slf14, a novel endophytic fungus producing huperzine a and hypocrellin a. Genome Announcements 2:1) e00011-1) e00014

Yew SM, Chan C, Soo-Hoo TS et al (2013) Draft genome sequence of dematiaceous coelomycete Pyrenochaeta sp. strain UM 256, isolated from skin scraping. Genome Announcements 1(3):e00158-e00113

Zdobnov EM, Tegenfeldt F, Kuznetsov D et al (2016) OrthoDB v9. 1: cataloging evolutionary and functional annotations for animal, fungal, plant, archaeal, bacterial and viral orthologs. Nucleic Acids Research 45:D744-D749

Zeiner CA, Purvine SO, Zink EM et al (2016) Comparative analysis of secretome profiles of manganese (II)-oxidizing ascomycete fungi. PLoS One 11(7):e0157844

Zeng F, Wang C, Zhang G, Wei J, Bradley CA, Ming R (2017) Draft genome sequence of Cercospora sojina isolate S9, a fungus causing frogeye leaf spot (FLS) disease of soybean. Genomics Data 12:79-80

Zhang C, Sayyari E, Mirarab S (2017) ASTRAL-III: increased scalability and impacts of contracting low support branches. In: RECOMB international workshop on comparative genomics, pp 53-75

\section{Publisher's Note}

Springer Nature remains neutral with regard to jurisdictional claims in published maps and institutional affiliations.

Ready to submit your research? Choose BMC and benefit from:

- fast, convenient online submission

- thorough peer review by experienced researchers in your field

- rapid publication on acceptance

- support for research data, including large and complex data types

- gold Open Access which fosters wider collaboration and increased citations

- maximum visibility for your research: over $100 \mathrm{M}$ website views per year

At BMC, research is always in progress.

Learn more biomedcentral.com/submissions 